(2) OPEN ACCESS
- Additional material is published online only. To view please visit the journal online (http://dx.doi.org/10.1136/ neurintsurg-2020-016412).

For numbered affiliations see end of article.

Correspondence to Dr Andreas Maximilian Frölich, Department of Diagnostic and Interventional Neuroradiology, University Medical Center Hamburg-Eppendorf, Hamburg 20246, Germany; a.froelich@ uke.de

Received 25 May 2020 Revised 28 July 2020 Accepted 28 July 2020 Published Online First 3 September 2020
Check for updates

(C) Author(s) (or their employer(s)) 2021. Re-use permitted under CC BY-NC. No commercial re-use. See rights and permissions. Published by BMJ.

To cite: Frölich AM, Kim W, Stribrny K, et al.

J Neurolntervent Surg

2021;13:722-726.

\title{
The novel Tenzing 7 delivery catheter designed to deliver intermediate catheters to the face of embolus without crossing: clinical performance predicted in anatomically challenging model
}

\author{
Andreas Maximilian Frölich (D) ,' Warren Kim, ${ }^{2}$ Knut Stribrny, ${ }^{1}$ Olav Jansen, ${ }^{3}$ \\ Markus Möhlenbruch, ${ }^{4}$ Istvan Szikora (D) , ${ }^{5}$ Fritz Wodarg (D) , ${ }^{6}$ Jens Fiehler, ${ }^{7}$ Kim Otto, $^{8}$ \\ Tony Chou, ${ }^{8}$ Jan Hendrik Buhk, ${ }^{1}$ Joey English ${ }^{2}$
}

\begin{abstract}
Background In large vessel occlusionstroke, navigation of aspiration catheters (AC) can be impeded by vessel tortuosity and the ophthalmic artery origin. A novel tapered delivery catheter was designed to facilitate delivery without disturbing the embolus. We assessed AC deliverability in vitro and validated the observations in a first-in-human experience.
\end{abstract}

Methods In a vascular model with three challenging craniocervical scenarios, two commercial AC were advanced from the carotid to the middle cerebral artery by four neurointerventionalists. Catheter deliverability with standard microwire and microcatheter (MC) combinations and the Tenzing 7 (T7) Delivery Catheter (Route 92 Medical, San Mateo, CA) were compared. Operators rated aspects of catheter deliverability on a 5-point scale. Results were compared with device delivery patterns at a neurovascular center before and after clinical introduction of $\mathrm{T} 7$.

Results In vitro, success rate and speed were higher with $\mathrm{T7}(96 \%$; mean $30 \pm 10$ s) than with MC $(65 \%$; $72 \pm 47 \mathrm{~s}, p<0.001$ each), with fewer interactions with the occlusion site (T7: $54 \%$ vs MC: $77 \%, \mathrm{p}=0.004$ ). T7 received superior ratings regarding carotid artery deflection (T7: 2, IQR1-3 vs MC: 3, IQR2-3, p<0.001), guide catheter pushback (T7: 2, IQR1-3 vs MC: 3, IQR3$3, p<0.001)$ and ophthalmic artery passage (T7: 1.5, IQR1-2 vs MC: 4, IQR3-5, $\mathrm{p}<0.001$ ). Before introduction of $\mathrm{T7}$ at a single center, delivery of $\mathrm{AC}$ to a large vessel occlusion without crossing was achieved in $15 / 123$ cases $(12 \%)$. With $T 7$, this rate was $28 / 31$ patients $(90.3 \%)$. Conclusion Compared with microcatheter and microwire combinations, $\mathrm{T7}$ improves aspiration catheter delivery in vitro, minimizing the need to cross the occlusion. Initial clinical experience appears to validate the model's observations.

\section{INTRODUCTION}

In acute ischemic stroke with intracranial large vessel occlusion, mechanical thrombectomy is the established, effective treatment option. ${ }^{1-7}$ Common endovascular technique entails use of a stentretriever device for removal of the intracranial thrombus. Alternatively, a direct aspiration firstpass technique (ADAPT) with large-bore aspiration catheters can be utilized, ${ }^{8}$ with results from two randomized trials suggesting efficacy comparable to stent retrievers. ${ }^{9} 10$

Navigation of catheters can be impeded by vessel tortuosity and parent artery branching (eg, the ophthalmic artery origin ${ }^{11}{ }^{12}$ ), a challenge which is particularly relevant for large-bore aspiration catheters, which may be less easily deliverable to the intracranial vessels than smaller microcatheters. Furthermore, operators may wish to avoid crossing the occlusion site as this may potentially cause thrombus fragmentation and embolization as well as frank vessel perforation. Novel, tapered delivery assist catheters have been designed to facilitate delivery of large-bore catheters to the intracranial circulation. To test whether such devices facilitate navigation, we assessed aspiration catheter delivery in several patient-specific in vitro models using a variety of different catheter combinations. We then compared these laboratory observations with clinical practice patterns at a single neurovascular center before and after introduction of such a delivery assist catheter.

\section{METHODS}

\section{In vitro experiments}

A previously developed flow model ${ }^{13}$ was modified to replicate an acute stroke intervention vascular access route from the femoral to the middle cerebral artery. A commercially available silicone model was used to replicate the femoral and thoracoabdominal vasculature (Neuro Testing Model NTM00V02, United Biologics, Santa Ana, CA). The cervical and intracranial vasculature was replicated with custom-made hollow models obtained by additive manufacturing using a commercially available laser stereolithography machine (Form 2, Formlabs, Somerville, MA, USA). Models were constructed based on anonymized imaging data (institutional review board approval obtained from Ethikkommission der Ärztekammer Hamburg, \#WF-034/14) and fabricated using a semi-elastic material (flexible resin, Formlabs, Somerville, MA). Different degrees of tortuosity were manually introduced in the digital cervical carotid artery models before manufacturing. Three different scenarios were realized 


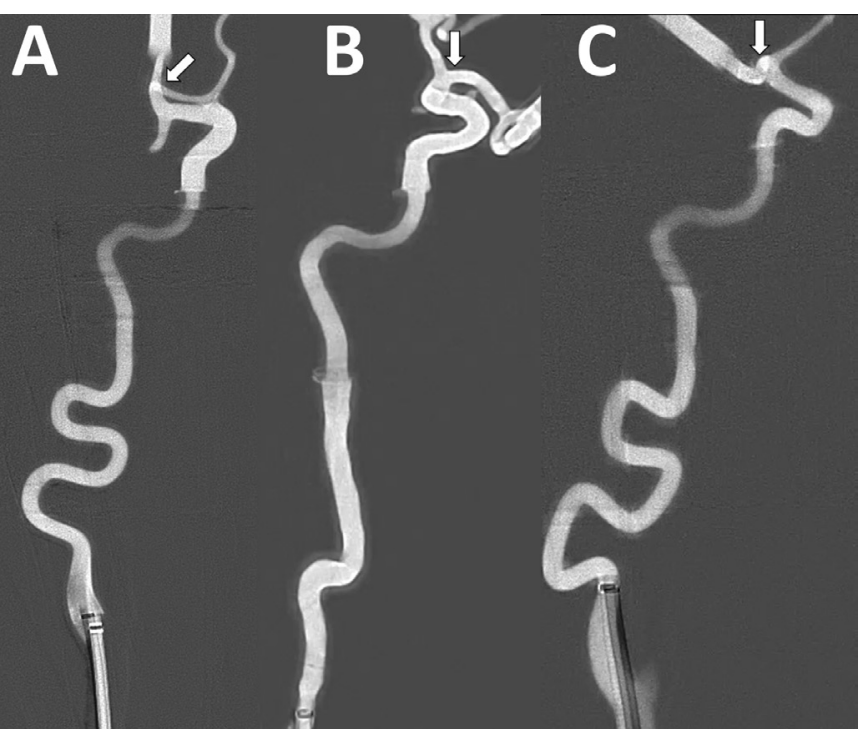

Figure 1 Model geometries. Panels A, B and C demonstrate fluoroscopic "roadmap" recordings from models 1, 2 and 3 with the guiding sheath in place. White arrows mark the proximal M1 segment as the target vessel for catheter delivery.

(figure 1): model 1 consisted of a curved cervical internal carotid artery with a moderately tortuous carotid siphon, model 2 consisted of a slightly tortuous cervical carotid artery and a severely tortuous siphon, while model 3 had a severely tortuous cervical carotid artery as well as a severely tortuous carotid

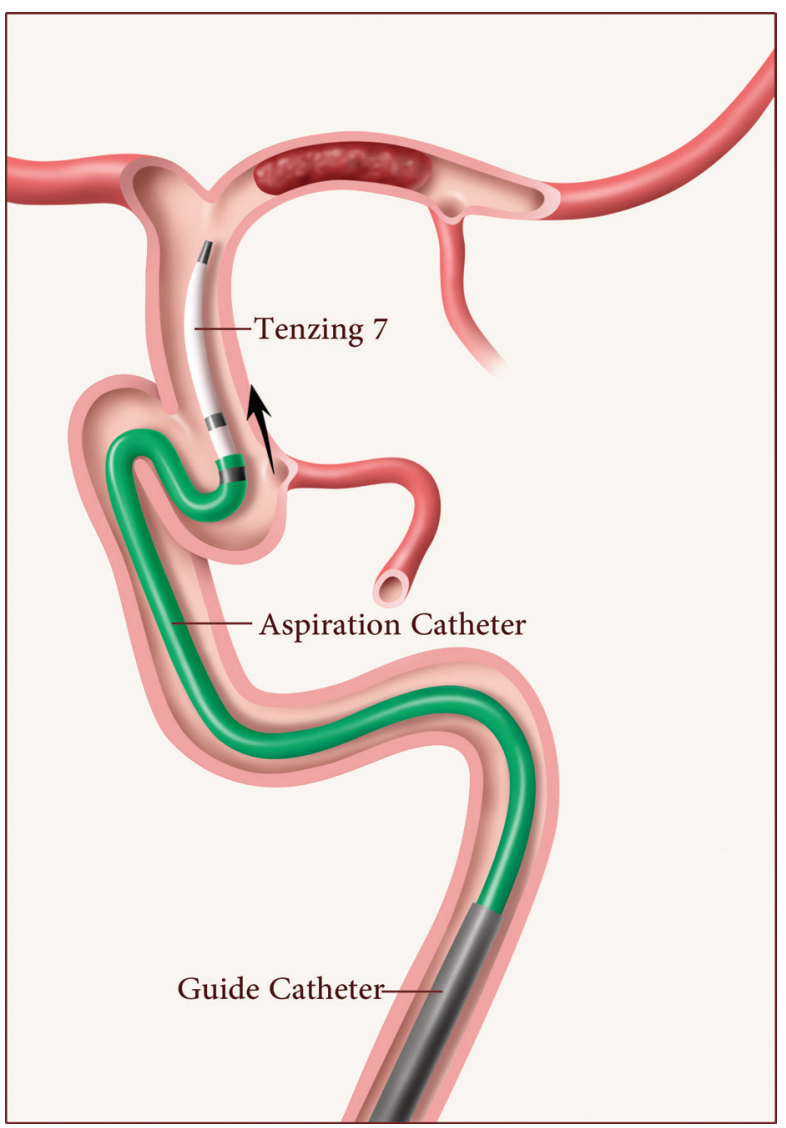

Figure 2 T7 design. The figure illustrates the conical tapered tip of T7 in combination with an aspiration catheter.
Table 1 Catheter combinations

\begin{tabular}{lll}
\hline Combination \# & Aspiration catheter & Microcatheter \\
\hline 1 & Sofia Plus* & Headway $2{ }^{*}$ \\
2 & Sofia Plus* & Tenzing $7 \dagger$ \\
3 & Ace $68 \ddagger$ & 3 MAX $\dagger$ \\
4 & Ace 68 & Tenzing 7† \\
\hline
\end{tabular}

${ }^{*}$ Microvention/Terumo, Irvine, CA.

tRoute 92 Medical, San Mateo, CA.

¥Penumbra Inc, Alameda, CA.

siphon. Models 1 and 2 were intended to present a moderate challenge to navigation, and model 3 a severe challenge. The models were perfused with water and $0.5 \%$ baby shampoo at $37^{\circ} \mathrm{C}$ under pulsatile flow $(1 \mathrm{~Hz}, 200 \mathrm{~mL} / \mathrm{min})$. The ostium and course of the ophthalmic artery and the posterior communicating artery were reproduced for approximately $1 \mathrm{~cm}$ in the models. Fluid outlets were connected to the middle and anterior cerebral artery only.

The Tenzing 7 (T7, Route 92 Medical, San Mateo, CA) is a novel delivery catheter specifically designed to deliver large bore catheters. The T7 has a smooth, flexible, conical tapered tip that reduces the ledge between it and catheters with an inner diameter between $0.068-0.072$ inch. T7 can be used with microwires up to 0.016 inch in diameter. See figure 2 and online supplementary for device schematics. T7 was assessed in combination with two commercially available aspiration catheters (Sofia Plus, Microvention/Terumo, Irvine, CA; and ACE68, Penumbra, Alameda, CA). For comparison, these aspiration catheters were delivered with microcatheters manufactured by the respective companies (Headway 21, Microvention/Terumo, Irvine, CA; and $3 \mathrm{MAX}$, Penumbra, Alameda, CA). Table 1 summarizes all employed combinations. All catheters were advanced through a commercial 0.088 inch sheath placed at the origin of the internal carotid artery. A 0.014 inch guidewire was employed; its use with T7 was optional at the discretion of the operators. T7 can be delivered as a unit together with the aspiration catheter or independently with step-by-step, alternating advancement of the T7 and aspiration catheter; operators were free to choose the desired technique.

For each catheter setup, four experienced neurointerventionalists (each with $>10$ years' experience as an independent operator) performed three attempts at delivering the aspiration catheter from the sheath to the proximal M1 segment of the middle cerebral artery under fluoroscopy and roadmap-guidance. Operators were instructed to proceed as per their clinical routine. After each attempt, operators rated carotid artery deflection, guide catheter pushback and difficulty passing the ophthalmic artery origin on a 5 -point scale ( $1=$ none, $5=$ most severe). Complete fluoroscopy was recorded during the experiments.

Procedural characteristics were assessed on fluoroscopy recordings and included time to successfully reach the occlusion site, time from first interaction with the ophthalmic artery to passing the ophthalmic artery with the aspiration catheter, any interaction of catheters or wires with the occlusion site, and maximum length of guidewire or microcatheter advancement beyond the occlusion site $(\mathrm{mm})$.

\section{Clinical data}

From a prospectively maintained database of acute stroke patients at Sutter Health California Pacific Medical Center (San Francisco, CA) and Mills-Peninsula Medical Center (Burlingame, CA), procedural characteristics before and after introduction of 


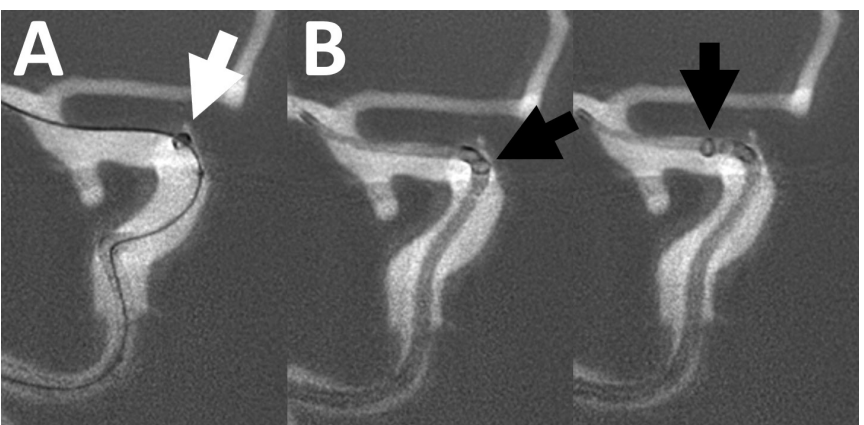

Figure 3 Crossing the ophthalmic artery origin. Fluoroscopy illustrates the "ledge effect" on attempted crossing of the ophthalmic artery. (A) With a microcatheter and wire technique, the aspiration catheter may fail to pass due to engaging with the ophthalmic artery ostium (white arrow). (B) The conical shape of the T7 delivery catheter can overcome the ledge effect, allowing the aspiration catheter tip (black arrow) to advance.

the T7 were analyzed under local investigational review board approval. Variables assessed included type of aspiration and microcatheter, the ability to deliver the catheter without crossing the occlusion site (WOX) with an MW or MC, and use of a stent retriever (which requires first crossing the occlusion with an MW and MC).

\section{Statistical analysis}

Continuous variables were compared using independent samples Student's t-test for two samples and the analysis of variance (ANOVA) for comparisons across operators and models. Proportions were compared with the $\chi^{2}$ test. Ordinal variables were compared using the Mann-Whitney U test. All tests were done using MedCalc (version 19.1.5, MedCalc bv, Ostend, Belgium). Data (statistical data and in vitro video files) are available on reasonable request through our institution's contact for data sharing (nrad@ uke.de).

\section{RESULTS}

\section{In vitro experiments}

A total of 138 out of 144 planned delivery attempts were successfully recorded. The target vessel was reached significantly more often with T7 (96\%) than with MC $(65 \%, \mathrm{p}<0.001)$. Failed attempts were mostly due to inability to advance the aspiration catheter past the ophthalmic artery origin caused by a "ledge effect" (figure 3, online supplementary video), that is, the aspiration catheter partly

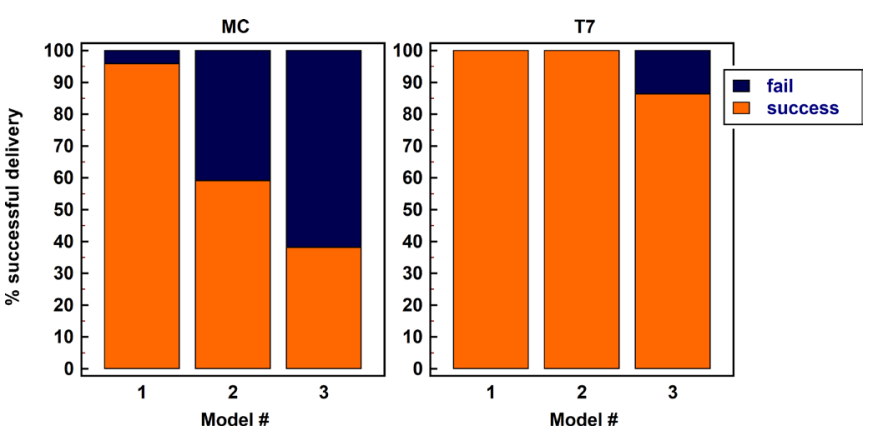

Figure 4 Delivery success rate. Stacked bar charts display the proportion of successful delivery attempts of aspiration catheters to the target vessel according to model geometry and delivery technique. MC, microcatheter technique; T7, Tenzing 7 catheter technique. engaging the ophthalmic artery origin (23 out of 27 failed attempts, $85 \%)$. On successful attempts $(\mathrm{n}=111)$, catheter delivery was faster with T7 (mean $30 \pm 10 \mathrm{~s})$ than with $\mathrm{MC}(72 \pm 47 \mathrm{~s}, \mathrm{p}<0.001)$, and time from first interaction with the ophthalmic artery to passing of the ophthalmic artery was significantly shorter with T7 (mean $2 \pm 3 \mathrm{~s})$ than with $\mathrm{MC}(21 \pm 25 \mathrm{~s}, \mathrm{p}<0.001)$.

Comparing the different operators, no significant differences were found in terms of delivery speed (mean \pm SD for operators 1 to $4: 50 \pm 60 \mathrm{~s}, 53 \pm 31 \mathrm{~s}, 44 \pm 25 \mathrm{~s}, 40 \pm 18 \mathrm{~s} ; \mathrm{p}=0.58$ ). With a total of three failed attempts, there was no significant difference in success rate between operators with T7 $(p=0.23)$. Among a total of 24 failed attempts with MC, we observed differences in success rate between operators $(\mathrm{p}=0.024)$, with operator 3 having a higher success rate than operator 4 on pair-wise comparison $(89 \%$ vs $41 \%, p=0.005)$. Comparing the different models, failure to reach the target vessel was increasingly observed going from model 1 to models 2 and 3 with the MC technique (figure 4). With T7, all delivery attempts in models 1 and 2 were successful. Catheter delivery was significantly faster in models 1 and 2 versus model 3 (mean $52 \pm 50$ s for model $1,46 \pm 29$ s for model $2,73 \pm 40$ s for model $3 ; \mathrm{p}=0.006)$.

There were fewer instances of interaction with the occlusion site with T7 (54\%) than with MC (77\%, p=0.004). The maximum length of guidewire or microcatheter advancement beyond the occlusion site was shorter for T7 (mean $5 \pm 6 \mathrm{~mm}$ ) than for MC $(15 \pm 15 \mathrm{~mm}, \mathrm{p}<0.001)$.

Finally, operators assigned superior ratings to $\mathrm{T} 7$ than to $\mathrm{MC}$ regarding carotid artery deflection (2, IQR1-3 vs 3, IQR2-3, $\mathrm{p}<0.001)$, guide catheter pushback $(2, \mathrm{IQR} 1-3$ vs 3, IQR3-3, $\mathrm{p}<0.001)$ and ophthalmic artery origin passage $(1.5$, IQR1-2 vs 4, IQR3-5, p <0.001).

\section{Clinical practice patterns}

In 2018, before the clinical introduction of T7, a large bore AC for first pass thrombectomy in the anterior circulation was delivered without a stent retriever in only 15 of 123 cases (12\%), none of which were done WOX. In the other $88 \%$ of these cases, it was necessary to cross the occlusion with an MW and MC for deployment of a stent retriever, after which the AC was railed up to the face of the occlusion. With T7, successful delivery of conventional aspiration catheters (REACT 071 (Medtronic Neurovascular, Irvine, CA), 6F Sofia/Sofia Flow Plus (Microvention/Terumo, Irvine, CA), CAT 7 (Stryker Neurovascular, Fremont, CA)) was achieved in $90.3 \%(28 / 31)$ without a stent retriever. In all cases, the T7 was first used without a microwire and delivered the large bore catheter WOX in $71 \%(22 / 31)$ of cases. In 29\% (9/31) of cases, a microwire was used to redirect the $\mathrm{T} 7$ from a branch in the anterior circulation (ophthalmic artery, fetal posterior communicating artery or A1 segment of anterior communicating artery), without crossing the occlusion with either the MW or the T7. In $9.7 \%$ (3/31) of cases, it was necessary to deploy a stent retriever across the occlusion to facilitate aspiration catheter delivery. There were no observed episodes of dissection, thrombus or perforation in this case series with first pass delivery with the T7 delivery catheter.

\section{DISCUSSION}

This study demonstrates that a dedicated delivery catheter facilitates advancement of large-bore aspiration catheters to the middle cerebral artery in vitro, particularly in challenging anatomy without crossing the embolus. Inability to pass the ophthalmic artery was the primary reason for failed delivery attempts with $\mathrm{MC}$, and differences in time spent traversing the ophthalmic artery origin accounted for approximately half of the total difference in delivery speed between MC and T7. Catheter deliverability was improved 
with $\mathrm{T} 7$ across all four experienced interventionalists, suggesting that the effect is not operator-dependent, although we did observe some differences in baseline operator success rate with the conventional MC method. Differences in success rate and speed between $\mathrm{MC}$ and $\mathrm{T} 7$ were most pronounced in the anatomically more challenging models. Recent data suggest that failed thrombectomy can be attributed to the inability to reach the occlusion site in more than $20 \%$ of cases. ${ }^{12}$ Thus, facilitation and acceleration of catheter delivery may be a valuable target in order to contribute to an increased success rate of thrombectomy. Importantly, this applies to both contact aspiration as well as to a variety of combined techniques utilizing stent retrievers together with an aspiration catheter.

Currently, there is limited evidence to guide the choice between contact aspiration and stent-retriever based thrombectomy, in part because of a lack of robust randomized data for contact aspiration versus intravenous tissue plasminogen activator (iv tPA). ${ }^{14}$ The ASTER and COMPASS trial results ${ }^{9}{ }^{10}$ suggest that contact aspiration may be a viable alternative to the use of stent retrievers in select populations. Interestingly, in the COMPASS trial, a slightly higher proportion of patients achieved Thrombolysis in Cerebral Infarction (TICI) 3 revascularization within $45 \mathrm{~min}$ in the aspiration group (34\%) than in the primary stent retriever group (23\%). It is quite conceivable that the efficacy of both contact aspiration as well as the combined techniques can be improved by larger aspiration catheters (a catheter with an inner diameter of 0.068 inch was most commonly used in COMPASS), as has been shown in theoretical modeling and animal experiments. ${ }^{15}$ For larger catheters, deliverability may be even more challenging, emphasizing the potential utility of specialized delivery catheters. Other devices have been designed to serve a similar purpose, including the Wedge catheter (Microvention/Terumo, Irvine, CA) and the AXS Offset (Stryker Neurovascular, Fremont, CA). These catheters have been adapted from a standard microcatheter as a constructional basis. The Tenzing device was designed with purely the delivery of the intermediate catheter in mind. Competitive delivery devices were not assessed in the present study.

In vitro, T7 decreased the need for any interaction with the occlusion and also reduced the length of MC or MW advancement beyond the occlusion site. This may help to further refine the technique of contact aspiration. A possible advantage of contact aspiration is the ability to remove clot without crossing the occlusion site with a microwire or microcatheter, an advantage that only holds true if the aspiration catheter can be delivered without crossing the embolus with an MC or MW or even interacting with it. Potentially, crossing the occlusion may lead to thrombus fragmentation and distal embolization; it may also increase the risk for vessel perforation. On the other hand, in vitro data suggest that contact aspiration may conversely increase the risk for thrombus fragmentation compared with stent-retrievers, ${ }^{16}$ although the aspiration catheter employed in that study was comparably small (0.054 inch distal inner diameter). Future analyses are required to determine whether the advantage of not crossing the occlusion site could outweigh potential risks of clot fragmentation.

The present laboratory findings are reflected by clinical practice patterns observed after introduction of $\mathrm{T} 7$ at a large interventional stroke center, which saw a ready adoption of the new device with high rates of deliverability. Interestingly, crossing the clot was even less frequently necessary clinically than in vitro. This might be explained by the very tortuous anatomy of the in vitro models employed, requiring more support for catheter advancement. Furthermore, crossing the occlusion site in vitro was assessed by objective analysis of fluoroscopy recordings, revealing also minimal interactions of the T7 with the occlusion site. In the clinical series these data were operator-reported, creating a potential for bias.
Interestingly, Pfaff et al report favorable delivery characteristics of large-bore aspiration catheters in acute stroke patients with the AXS Offset. ${ }^{17}$ Taken together, these observations support the use of in vitro models as a platform to guide catheter and device development as well as assessment. If more data on the anatomy of clinically challenging cases could be gathered, it might even be conceivable to establish a standardized in vitro model serving as a benchmark environment for device development, testing and, potentially, certification.

The study has several limitations. Tortuosity of the cervical internal carotid arteries was manually introduced and may therefore not ideally represent the anatomy encountered during stroke intervention. We did not include $360^{\circ}$ curves or vessel stenosis. In our opinion, however, the geometries utilized adequately provided navigational challenges at least similar to what can be encountered clinically. As the ophthalmic and posterior communicating arteries were not connected to a fluid outlet, they were only partly visualized on fluoroscopy. The starting position for each attempt had the sheath already placed at the carotid bifurcation, precluding us from assessing the overall procedure time (ie, from femoral puncture to closure). The number of attempts per operator was limited. Furthermore, we did not introduce clots into the model and flow conditions were thus different from those encountered clinically with a large vessel occlusion. We chose to do this in order to standardize flow conditions across all experiments, as artificial clots can be difficult to position in precisely identical locations across a multitude of attempts. The subjective, semi-quantitative evaluation of catheter behavior by the operators may be biased because the operators could not be blinded to the employed technique. Finally, as T7 is designed to deliver an aspiration catheter WOX, this may have created bias in the clinical series towards preferential use of contact aspiration as the primary approach, limiting the validity of our observations.

\section{CONCLUSION}

Compared with microcatheter and microwire combinations, the T7 delivery catheter significantly facilitates aspiration catheter delivery to the target vessel in vitro and reduces interaction with the occlusion site. These laboratory findings are reflected by practice patterns at a large interventional stroke center after clinical introduction of $\mathrm{T} 7$, highlighting the potential of in vitro testing for device development and assessment.

\section{Author affiliations}

${ }^{1}$ Department of Diagnostic and Interventional Neuroradiology, University Medical Center Hamburg-Eppendorf, Hamburg, Germany

${ }^{2}$ Radiology, California Pacific Medical Center, San Francisco, California, USA ${ }^{3}$ Department of Radiology and Neuroradiology, University Medical Center SchleswigHolstein Campus Kiel, Kiel, Schleswig-Holstein, Germany

${ }^{4}$ Neuroradiology, University Hospital Heidelberg, Heidelberg, Baden-Württemberg, Germany

${ }^{5}$ Neurointerventions, National Institute of Neurosciences, Budapest, Hungary ${ }^{6}$ Department of Radiology and Neuroradiology, Universitatsklinikum SchleswigHolstein Campus Kiel, Kiel, Germany

${ }^{7}$ Department of Neuroradiology, University Medical Center Hamburg-Eppendorf, Hamburg, Hamburg, Germany

${ }^{8}$ Route 92 Medical, Inc, San Mateo, California, USA

Contributors JF and TC handled funding and supervision. JE, AMF, OJ, MM, IS, $F W, K O, J H B$ and TC performed the experiments. DE, AMF, WK and KS analyzed the data. AMF, JE and WK wrote the manuscript. All authors read and corrected the manuscript.

Funding This work was supported by a grant from the German Ministry for Education \& Research (BMBF grant \#031L0154B). The authors thank Philips Healthcare for the support and realization of the "Hermann Zeumer Research Laboratory" including a Philips Allura Clarity Angiography system.

Competing interests ROUTE 92 MEDICAL \& TENZING are registered trademarks of Route 92 Medical, Inc. T7 is a trademark of Route 92 Medical, Inc. Catheters 
and travel support for physicians participating in the laboratory experiments were supplied by Route 92 Medical, Inc.

Patient consent for publication Not required.

Provenance and peer review Not commissioned; externally peer reviewed.

Data availability statement Data are available upon reasonable request. Data (statistical data and in vitro video files) are available upon reasonable request through our institution's contact for data sharing (nrad@uke.de).

Open access This is an open access article distributed in accordance with the Creative Commons Attribution Non Commercial (CC BY-NC 4.0) license, which permits others to distribute, remix, adapt, build upon this work non-commercially, and license their derivative works on different terms, provided the original work is properly cited, appropriate credit is given, any changes made indicated, and the use is non-commercial. See: http://creativecommons.org/licenses/by-nc/4.0/.

\section{ORCID iDs}

Andreas Maximilian Frölich http://orcid.org/0000-0002-0804-1056

Istvan Szikora http://orcid.org/0000-0003-3730-3278

Fritz Wodarg http://orcid.org/0000-0003-1413-2699

\section{REFERENCES}

1 Albers GW, Marks MP, Kemp S, et al. Thrombectomy for stroke at 6 to 16 hours with selection by perfusion imaging. N Engl I Med 2018;378:708-18.

2 Berkhemer OA, Fransen PSS, Beumer D, et al. A randomized trial of intraarterial treatment for acute ischemic stroke. N Engl J Med 2015;372:11-20.

3 Campbell BCV, Mitchell PJ, Kleinig TJ, et al. Endovascular therapy for ischemic stroke with perfusion-imaging selection. N Eng/ J Med 2015;372:1009-18.

4 Goyal M, Demchuk AM, Menon BK, et al. Randomized assessment of rapid endovascular treatment of ischemic stroke. N Engl J Med 2015;372:1019-30.

5 Jovin TG, Chamorro A, Cobo E, et al. Thrombectomy within 8 hours after symptom onset in ischemic stroke. N Engl J Med 2015;372:2296-306.
6 Nogueira RG, Jadhav AP, Haussen DC, et al. Thrombectomy 6 to 24 hours after stroke with a mismatch between deficit and infarct. N Engl J Med 2018;378:11-21.

7 Saver JL, Goyal M, Bonafe A, et al. Stent-retriever thrombectomy after intravenous t-PA vs. t-PA alone in stroke. N Engl J Med 2015:372:2285-95.

8 Turk AS, Spiotta A, Frei D, et al. Initial clinical experience with the ADAPT technique: a direct aspiration first pass technique for stroke thrombectomy. J Neurointerv Surg 2014;6:231-7

9 Turk AS, Siddiqui A, Fifi JT, et al. Aspiration thrombectomy versus stent retriever thrombectomy as first-line approach for large vessel occlusion (COMPASS): a multicentre, randomised, open label, blinded outcome, non-inferiority trial. Lancet 2019;393:998-1008.

10 Lapergue B, Blanc R, Gory B, et al. Effect of endovascular contact aspiration vs stent retriever on revascularization in patients with acute ischemic stroke and large vessel occlusion: the ASTER randomized clinical trial. JAMA 2017;318:443-52.

11 Lin L-M, Colby GP, Jiang B, et al. Classification of cavernous internal carotid artery tortuosity: a predictor of procedural complexity in pipeline embolization. J Neurointerv Surg 2015;7:628-33.

12 Leischner $\mathrm{H}$, Flottmann $\mathrm{F}$, Hanning $\mathrm{U}$, et al. Reasons for failed endovascular recanalization attempts in stroke patients. J Neurointerv Surg 2019:11:439-42.

13 Nawka MT, Spallek J, Kuhl J, et al. Evaluation of a modular in vitro neurovascular procedure simulation for intracranial aneurysm embolization. J Neurointerv Surg 2020;12:214-9.

14 Andersson T, Wiesmann M, Nikoubashman 0, et al. The aspirations of direct aspiration for thrombectomy in ischemic stroke: a critical analysis. J Stroke 2019:21:2-9.

15 Nikoubashman 0 , Nikoubashman $A$, Büsen $M$, et al. Necessary catheter diameters for mechanical thrombectomy with ADAPT. AJNR Am J Neuroradiol 2017:38:2277-81.

16 Chueh J-Y, Puri AS, Wakhloo AK, et al. Risk of distal embolization with stent retriever thrombectomy and ADAPT. J Neurointerv Surg 2016;8:197-202.

17 Pfaff JAR, Siekmann R, Shah YP, et al. Delivery assist catheters: a new device class and initial experience in mechanical thrombectomy in acute ischemic stroke patients. Clin Neuroradiol 2019:29:661-7. 